\title{
The effects of stimulus structure and familiarity on same-different comparison*
}

\author{
HOWARD S. HOCK ${ }^{+}$ \\ Florida Atlantic University, Boca Raton, Florida $3 \hat{3} 432$
}

\begin{abstract}
It was hypothesized that performance in the same-different comparison task is based on two modes of processing: (1) structural processes that organize the detailed parts of a stimulus into a well-formed whole, and (2) analytic processes that decompose the stimulus into features. This hypothesis was supported, but with the unexpected finding of individual differences in the mode of processing underlying "same" responses. Those Ss in the "same" condition whose reaction times were faster for symmetrical than for asymmetrical patterns supported the hypothesis for structural processes. The remaining Ss in the "same" condition, as well as all the Ss in the "different" condition, were unaffected by symmetry. These Ss supported the hypothesis for analytic processes. Although familiarity effects were obtained for both structural and analytic Ss, the rotation of the familiar patterns into an unfamiliar orientation virtually eliminated familiarity effects for the structural Ss, but left them intact for analytic Ss.
\end{abstract}

In recent years, there has been extensive research using the same-different comparison task, which involves the measurement of reaction times for "same" and "different" responses to pairs of simultaneously (or sequentially) presented stimuli. For the most part, this research has been based on the assumption that feature analysis is the fundamental process underlying both "same" and "different" comparison. In his recent review of this literature, however, Nickerson (1972) discusses two problems which suggest that this assumption may be invalid.

The first problem is based on evidence that "same" and "different" responses are differentially affected by the same stimulus manipulations. On the one hand, only "different" reaction times are affected by the number of relevant stimulus attributes (Hawkins, 1969), the size of an irrelevant bracket (Krueger, 1970), and the number of letters being compared (Beller, 1970). On the other hand, only "same" reaction times are affected by the meaningfulness of letter trigrams and the orientation of familiar alphabet letters (Egeth \& Blecker, 1971). If the assumption that feature analysis underlies both "same" and "different" responses is valid, the evidence described above indicates that the features are being processed in a very different manner for the two kinds of responses.

The second problem involves the fact that "same" responses are often faster than "different" responses (e.g., Bamber, 1970). The latter finding is a source of difficulty for feature-analysis models because a correct

*This paper is based on a doctoral dissertation submitted to The Johns Hopkins University. October 1972. The invaluable assistance of Howard Egeth, the author's thesis advisor, is gratefully acknowledged. Thanks are also extended to Bert Green, William Bevan, Warren Torgerson, and John Jonides, whose suggestions were of great benefit to the author. The experimental research was supported by a contract between the Engineering Psychology Programs, Office of Naval Research, and The Johns Hopkins University.

$\div$ Requests for reprints should be sent to Howard S. Hock, Department of Psychology, Florida Atlantic University. Boca Raton, Florida 33432. "different" response can be based on the detection of any differentiating feature, while a correct "same" response must be the result of an exhaustive comparison of all the features of the stimuli. On this basis, "same" responses could be as fast as "different" responses, but they should never be faster.

Although it remained conceivable that a sufficiently elaborate feature-analysis model could account for all the discrepancies described above, the purpose of the research reported in this paper was to explore the possibility that two fundamentally different processes underlie "same" and "different" comparison.

\section{A Two-Process Hypothesis}

Since a "different" response can be based on the detection of a single differentiating feature, it was hypothesized that "different" comparison is based on analytic processes that decompose the stimulus information into features. This hypothesis was supported most directly by Hawkins's (1969) finding that the speed of "different" responses decreased as the number of features that differentiated pairs of "different" stimuli was increased. Since "same" responses require the comparison of all the information in each stimulus, it was hypothesized that "same" comparison involves a process of structuring the stimulus information into well-organized units, or chunks. The two-process hypothesis, then, was that "same" comparison is based on structural processes and "different" comparison on analytic processes.

An important qualification to this two-process model is that it does not require that "same" responses be faster than "different" responses. The latter would depend on the nature of the stimuli; the speed of "same" responses would be determined by how well each stimulus could be structured or organized into a whole, while the speed of "different" responses would depend on the number of features that differentiate the stimuli constituting "different" pairs (as well as the 
discriminability of these features). That is, the relative speeds of "same" and "different" responses would, according to the two-process hypothesis, depend on the "goodness of form" of each stimulus relative to the discriminability of the total set of stimuli (there was, however, no attempt to manipulate the relative speeds of "same" and "different" responses in the present study).

In order to examine the hypothesis that "same" and "different" responses are based on different processes, half the stimuli used in the present research were symmetrical and half were asymmetrical. The possibility that symmetry could function as a feature that would differentiate between two "different" stimuli was eliminated by designing the experiment so that: (1) all the symmetrical stimuli had the same axis of symmetry, and (2) the stimuli forming "different" pairs were either both symmetrical or both asymmetrical. In this way, differences in response time between pairs of symmetrical and pairs of asymmetrical stimuli could not be attributed to the use of symmetry as a response-relevant feature.

For "same" comparison, it was hypothesized that responses would be faster for pairs of identical symmetrical stimuli than for pairs of identical asymmetrical stimuli. This hypothesis was based on the assumption that: (1) the structural processes underlying "same" responses are concerned with the organization of the detailed parts of the stimulus into a well-formed whole, and (2) symmetry would facilitate structural processes, and thereby "same" Iesponses, by providing a simple rule for putting the detailed parts of a stimulus into a structured relationship. 1 Since "different" responses were hypothesized to depend on feature analysis and the experiment was designed so that symmetry was not a response-relevant feature, a difference in reaction time between pairs of symmetrical and pairs of asymmetrical stimuli was not predicted for "different" responses.

\section{Familiarity and the Two-Process Hypothesis}

If, as hypothesized, same-different comparison involves two kinds of processing, then evidence that past experience affects "same" and/or "different" responses would have important implications with respect to the kind of knowledge acquired for the familiar stimuli. If past experience influenced "same" comparison, it would imply that some kind of structural, or schematic, knowledge had been acquired for the familiar stimuli. If past experience influenced "different" comparison, it would imply that knowledge of distinctive features had been acquired for the familiar stimuli.

In a recent experiment using the same-different comparison task, Egeth and Blecker (1971) manipulated familiarity by rotating familiar alphabet letters into an unfamiliar orientation (in a given stimulus pair, both letters were always presented in the same orientation). Using a mixed sequence of familiar and rotated-familiar stimuli, Egeth and Blecker found that familiarity affected reaction times for "same" but not for "different" responses. This finding, in conjunction with the two-process hypothesis, implied that past experience influenced structural, but not analytic, processes in the same-different comparison task. Given the importance of this implication, it was necessary to determine whether Egeth and Blecker's results depended on their use of rotation as a manipulation of familiarity. To this end, the experiment reported in this paper was designed so that a more direct manipulation of familiarity could be introduced into the same-different comparison task. This was done by providing Ss with preliminary training at reproducing stimuli that were initially unfamiliar and designing the experiment so that the familiar (as a result of training) and unfamiliar (no training) stimuli were matched in complexity. The posttraining same-different comparison task then included pairs of familiar stimuli, pairs of unfamiliar stimuli, and pairs of rotated-familiar stimuli. If a difference in response time between the familiar and unfamiliar stimuli were obtained, it would provide direct evidence that past experience affects "same" and/or "different" comparison.

To summarize, it was predicted that symmetry would affect reaction times for "same" but not for "different" responses. This would support the hypothesis that "same" comparison is based on structural processes, while "different" comparison is based on analytic processes. Furthermore, with familiarity manipulated in a more direct manner than in Egeth and Blecker's experiment, familiarity effects were hypothesized for both "same" and "different" responses. Whereas a familiarity effect for "different" responses could be interpreted in terms of the acquisition of distinctive features, the manner in which familiarity might influence structural processes is essentially unknown. For this reason, there was no basis for predicting whether familiarity and symmetry would have independent, or interactive, effects on "same" responses. It was hoped that the data obtained in the present study, together with the results of future research, would provide more insight into the relationship between stimulus structure (e.g., symmetry) and familiarity.

\section{METHOD}

\section{Experimental Design}

The initial step in designing this experiment was to circumvent the problem of matching unfamiliar stimuli to stimuli that were preexperimentally familiar. This was accomplished by using stimuli that were initially unfamiliar and subdividing these stimuli into two sets (I and II) for assignment to the familiar (F) and unfamiliar (U) conditions. Those stimuli assigned to the familiar condition were presented during two preliminary training sessions, as well as the experimental session, while those assigned to the unfamiliar condition were presented only during the experimental session. Since the assignment of the two sets of stimuli (I and II) to the two familiarity conditions ( $F$ and $U$ ) was counterbalanced between groups of $S s$, each stimulus appeared in both the familiar and unfamiliar conditions and the familiar 
and unfamiliar stimuli were perfectly matched in complexity.

The stimuli used were dot patterns; half were symmetrical and half were asymmetrical. The partitioning of the patterns into two sets ( $\mathrm{I}$ and $\mathrm{II}$ ) for assignment to the familiarity conditions ( $\mathrm{F}$ and $U$ ), together with the further subdivision between symmetrical (S) and asymmetrical (A) patterns, resulted in the formation of four subsets of dot patterns. There were five different dot patterns in each subset. In addition to the familiar and unfamiliar condition, there was a third condition $\left(F_{R}\right)$, in which the patterns assigned to the familiar condition were rotated $180 \mathrm{deg}$. The experiment was therefore designed so that there were three levels of familiarity $\left(F, F_{R}, U\right)$ that were orthogonal with respect to two levels of symmetry $(S, A)$.

The main experimental task consisted of the same-different comparison of simultaneously presented dot patterns, with the reaction time of the "same" and "different" responses constituting the dependent variable of primary interest. The patterns that were paired together were always drawn from the same familiarity condition, and both patterns were either symmetrical or asymmetrical. For example, a symmetrical pattern assigned to the unfamiliar condition could only be paired with itself (to form a "same" pair) or with one of the other four symmetrical patterns assigned to the unfamiliar condition (to form a "different" pair). The experimental session consisted of a mixed sequence of 240 trials resulting from the six combinations of the familiarity $\left(F, F_{R}, U\right)$ and symmetry $(S, A)$ variables. There were, for example, a total of 40 trials consisting of symmetrical, unfamiliar stimuli. Twenty of these were "same" pairs, with each of the five symmetrical, unfamiliar patterns repeated in four "same" stimuli. The 20 "different" stimuli consisted of the pairwise combination of the five symmetrical, unfamiliar patterns. Each of these patterns appeared equally often in the left and right positions of the "different" stimuli.

Since it was hypothesized that "same" and "different" responses were based on different processes, it was desirable to keep the two responses as separate as possible. For this reason, two groups of Ss were assigned to independent "same" and "different" conditions. The Ss assigned to the "same" condition pressed the response button only when the two patterns were the "same." When the patterns were "different," they did not respond in any way. The reverse was the case for Ss assigned to the "different" condition.

\section{Stimuli}

\section{Pattern Construction}

There were eight dots in each pattern, with each dot centered in one of 25 possible positions in a $5 \times 5$ grid. The grid served only to locate the dots; no grid lines ever appeared in any of the stimuli. The dots, which were black, were placed on a white background with the distance between the centers of adjacent dots (in the same row or column) equal to iwice the dot diameter. A total of 32 patterns were constructed; half were symmetrical and half were asymmetrical. All the symmetrical patterns had their axis of symmetry extending from the upper left to the lower right corners of the $5 \times 5$ grid. 2 There were three restrictions on the patterns that were constructed: (1) at least one dot was present in each row and column of the pattern grid (following Garner \& Clement, 1963); (2) in order to minimize differences in complexity between the patterns, dots were never placed in three contiguous, linearly connected cells; and (3) an attempt was made to construct patterns that had distinctive forms or shapes.

\section{Pattern Selection}

In order to evaluate properly the experimental hypotheses formulated for this investigation, preliminary procedures were required to match the subsets of symmetrical and asymmetrical patterns with respect to preexperimental familiarity. similarity,

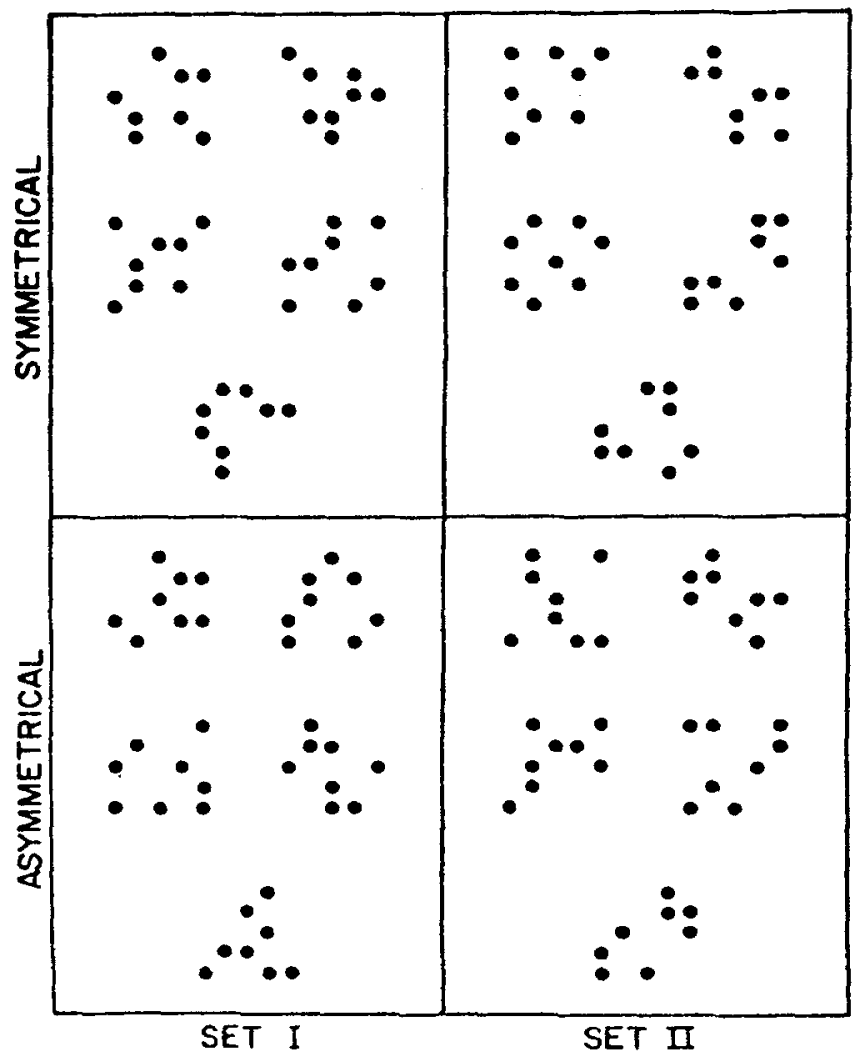

Fig. 1. The $\mathbf{2 0}$ dot patterns used in the experiment.

and central dispersion. These procedures, which are described in greater detail by Hock (1971), yielded the following measures for each pattern: (1) its preexperimental familiarity, as determined by the extent to which Ss agree on its name; (2) its similarity to the other symmetrical (or asymmetrical) patterns, as determined by both the overlap of dot locations and $S s^{\prime}$ ratings of similarity; and (3) its central dispersion, as determined by the average distance of the dots from the center of the pattern.

The symmetrical and asymmetrical patterns were matched with tespect to these measures by selecting 20 patterns from the original 32 . For the final 20 patterns, the 10 symmetrical and 10 asymmetrical patterns were, on the average, very closely matched in terms of preexperimental familiarity, central dispersion, and objectively measured similarity (overlap of dot locations). For subjective ratings of similarity, however, there was a small tendency for pairs of symmetrical patterns to be rated higher in similarity than pairs of asymmetrical patterns. The 20 patterns that were selected for use in this experiment are presented in Fig. 1.

\section{Procedure}

\section{Symmetry Sensitization}

As the result of a pilot study, it was found that Ss often failed to notice that many of the dot patterns were symmetrical. This may have been due to the atypical $45-$ deg tilt of the axis of symmetry. To rectify the problem, the following procedure was instituted as the first step in the experimental procedure.

The $S s$ were shown each of the 10 patterns that would later be presented for familiarization training, and the axis of symmetry was denoted for each of the five symmetrical patterns. All 10 patterns were then presented, one at a time, in randomized blocks of 10 , the $S$ s being required to report whether or not the 

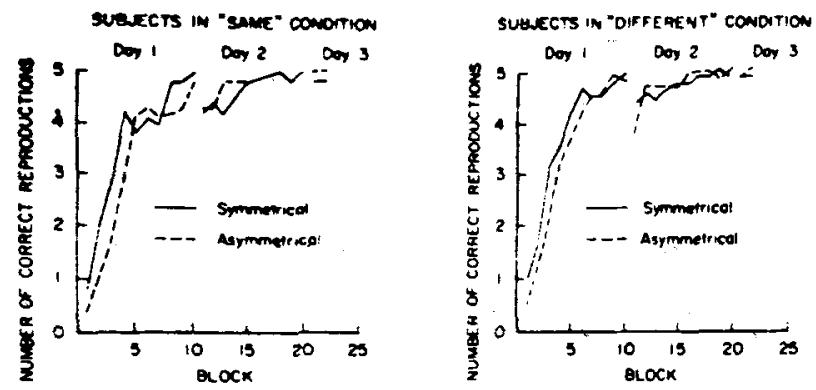

Fig. 2. Reproduction data. There were 10 trials in each block, 5 symmetrical and 5 asymmetrical.

exposed pattern was symmetrical. The stimuli were presented with a Carousel projector, and exposure times were controlled with a tachistoscopic shutter. The exposure duration was $2.0 \mathrm{sec}$ for the first block, with succeeding blocks of patterns exposed for $1.0,1.0,0.3,0.3,0.1$, and 0.1 sec.

\section{Familiarization Training}

The Ss were then familiarized with the same 10 dot patterns ( 5 symmetrical and 5 asymmetrical) during 2 days of training at reproducing them. Since the $S s$ had only to place dots on a blank $5 \times 5$ grid. the importance of artistic skill was minimized and the problem of objectively scoring the reproductions was eliminated.

The Ss were told that they would be seeing the same $: 10$ patterns that they had just judged for symmetry, that there were eight dots in each pattern, and that they were to learn to reproduce the patterns with perfect accuracy. They were not told that each row and column had at least one dot. A Carousel projector and tachistoscopic shutter was used to present each pattern for $3 \mathrm{sec}$. Following each presentation, the Ss were required to reproduce the pattern on a blank grid, guessing if they were unsure of the location of some of the dots. A correct version of the pattern (with grid lines) was provided after each incorrect reproduction. There were 10 blocks of trials, each block consisting of 10 trials (one for each pattern) presented in a different random sequence. Before starting the last block of trials, the $S$ s were reminded to be as accurate as possible in their reproductions. Only those $S$ s who achieved a reasonable level of familiarity with the patterns, as indicated by a minimum of 9 out of 10 correct reproductions on the last block of trials, were retained in the experiment. The entire procedure for Day 1 lasted about $55 \mathrm{~min}$.

The training session for Day 2, which lasted about $25 \mathrm{~min}$, consisted of 10 more blocks of reproductions. The patterns were now presented for a duration of 1 sec rather than 3 sec. Day 3 began with two blocks of reproductions, the first at a 1-sec and the second at a $0.3-\mathrm{sec}$ exposure duration. The $\mathrm{Ss}$ did not receive corrective feedback on any of the reproduction trials of Day 3. The experimental session followed these last two blocks of reproductions.

\section{Experimental Session}

The Ss were told that they would be seeing pairs of dot patterns, one alongside of the other, that were: (1) pairs of patterns they had practiced reproducing during the previous 2 days, (2) the same patterns, but turned upside-down, or (3) pairs of new patterns they hadn't seen before. They were told that half the new patterns would be symmetrical, with the same axis of symmetry as the old patterns. It was also pointed out that the patterns were the same for half the stimuli and different for the other half and that there would never be a stimulus for which the same pattern was presented in two different orientations.

Following a warning chime that preceded each stimulus by
$1 \mathrm{sec}$, a pair of dot patterns was simultaneously presented for a period of $3 \mathrm{sec}$, unless the $S$ responded, which immediately terminated the exposure. The stimuli were displayed on a screen by a Carousel projector, and the exposure was controlled by a tachistoscopic shutter. The stimuli were presented in a normally lighted room, without pre- or postpresentation masking stimuli. The visual angle for each pattern was $1.2 \mathrm{deg}$, and the overall visual angle across the pair of patterns was $3.5 \mathrm{deg}$.

The Ss were given a box with a single button that was to be pressed with a finger of their preferred hand whenever the pair' of patterns was the "same" (or "different," depending on the condition to which they were assigned). Otherwise, they were not to respond. Instructions to respond as quickly as possible within the context of keeping errors to a minimum served to stress both speed and accuracy.

Ten practice trials with pairs of alphabet letters were followed by 40 practice trials with pairs of dot patterns. The latter stimuli, which were repeated as the last 40 trials of the experimental sequence, consisted of patterns drawn from all the familiarity and symmetry conditions. The Ss were not told that these were practice trials. Rest breaks of about 1 min were taken after every 40 trials, and a break of about 3 min was taken after 120 trials. The entire procedure for Day 3 took about $45 \mathrm{~min}$.

\section{Subjects}

A total of 24 undergraduate students at Johns Hopkins University participated in the full 3 days of experimentation. These Ss received payment of $\$ 5.00$. Another $13 \mathrm{Ss}$ failed to pass the criterion set at the end of the first day and were paid $\$ 1.50$ for their participation.

\section{RESULTS}

\section{Reproduction Task}

The Ss' reproductions were scored on an all-or-none basis; they were either right or wrong. Since each block of trials consisted of five symmetrical and five asymmetrical patterns, the maximum score for each kind of pattern was 5. Performance on the reproduction task for the training sessions of Days 1 and 2, as well as the two blocks of reproduction trials that preceded the experimental session of Day 3, is summarized in Fig. 2 . Thirteen of 37 Ss failed to pass the criterion of 9 out of 10 correct on the last block of trials on Day 1. The data for these Ss, who were dropped from the experiment, are not included in Fig. 2.

The initial superiority in reproducing the symmetrical patterns, which disappeared by the end of Day 1 , was in agreement with the results obtained by Attneave (1955). The possibility that a residual difference between the patterns was obscured by a ceiling effect at the end of Day 1 was supported by the reappearance of the symmetry difference at the start of Day 2 . As a result of the overtraining provided on Day 2, however, the patterns were sufficiently familiar for any residual difference between the symmetrical and asymmetrical patterns to be undetectable in the reproduction trials of Day 3. When the number of correct reproductions was summed over all trials, the difference in performance between the symmetrical and asymmetrical patterns was insignificant for the Ss assigned to the "same" condition 
$[t(11)=1.79, p>05]$ and for the Ss assigned to the "different" condition $[t(11)=1.18, p>.05]$.

\section{Same-Different Comparison Task}

The mean response times and errors for the Ss in the "same" and "different" conditions are presented in Table 1. Since the experiment was concerned with differences in mode of processing for "same" and "different" responses, and not their relative speeds, the data for the "same" and "different" conditions (involving separate groups of Ss) was analyzed separately.

For the Ss in the "same" condition, the effect of familiarity $\left(F, F_{R}, U\right)$ was significant $[F(2,16)=5.24$, $\mathrm{p}<.05$ ], the effect of symmetry (S, A) was insignificant $[F(1,8)=2.21, p>.05]$, and the interaction between familiarity and symmetry was insignificant $[F(2,16)<1.0]$. For the Ss in the "different" condition, the effects of familiarity $[F(2,16)=2.40]$, symmetry $[F(1,8)<1.0]$, and the Familiarity by Symmetry interaction $[F(2,16)=2.55]$ were all insignificant at the .05 level.

Paired comparisons indicated that the difference in reaction time between the familiar and unfamiliar patterns ( $F$ vs $U$ ), the most direct determination of whether past experience influences performance on the same-different comparison task, was significant for both "same" responses $[\mathrm{t}(11)=2.89, \mathrm{p}<.05]$ and "different" responses $[\mathrm{t}(11)=2.69, \mathrm{p}<.05]$. Furthermore, Egeth and Blecker's finding that rotating familiar stimuli into an unfamiliar orientation affected only "same" responses was replicated. That is, $\left(F_{\mathbf{R}}-F\right)$ was significant $[t(11)=2.65, p<.05]$ for "same" responses but was insignificant $[t(11)<1.0]$ for "different" responses.

\section{Individual Differences}

A possible explanation for the insignificance of the

Table 1

Mean Reaction Times (in Milliseconds) and Total Errors for "Same" and "Different" Conditions

\begin{tabular}{|c|c|c|c|c|c|c|}
\hline & \multicolumn{2}{|c|}{ Mean RT } & \multirow[b]{2}{*}{$(A+S) / 2$} & \multirow[b]{2}{*}{$A-S$} & \multicolumn{2}{|c|}{ Total Errors } \\
\hline & $S$ & $\mathbf{A}$ & & & $\mathbf{S}$ & $\mathbf{A}$ \\
\hline & & & "Same" C & ndition & & \\
\hline \multirow[t]{3}{*}{$\begin{array}{l}F_{R} \\
F_{R}\end{array}$} & $\begin{array}{l}863 \\
887 \\
941\end{array}$ & $\begin{array}{l}899 \\
942 \\
975\end{array}$ & $\begin{array}{l}881 \\
915 \\
958\end{array}$ & $\begin{array}{r}+36 \\
+55 \\
+34\end{array}$ & $\begin{array}{c}11 \\
7 * \\
8\end{array}$ & $\begin{array}{r}15 \\
10 \\
2\end{array}$ \\
\hline & 897 & 939 & 918 & +42 & & \\
\hline & \multicolumn{6}{|c|}{ "Different" Condition } \\
\hline \multirow[t]{2}{*}{$\begin{array}{l}F \\
F_{R} \\
U\end{array}$} & $\begin{array}{l}875 \\
894 \\
920\end{array}$ & $\begin{array}{l}899 \\
894 \\
904\end{array}$ & $\begin{array}{l}887 \\
894 \\
912\end{array}$ & $\begin{array}{r}+24 \\
0 \\
-16\end{array}$ & $\begin{array}{l}11^{*} \\
2 \\
5^{* *}\end{array}$ & $\begin{array}{c}8 \\
20^{* *} \\
5\end{array}$ \\
\hline & 896 & 899 & 898 & +3 & & \\
\hline
\end{tabular}

*Indicates that an error of omission is included. The overall error rate was $1.8 \%$ for both the "same" and "different" conditions.

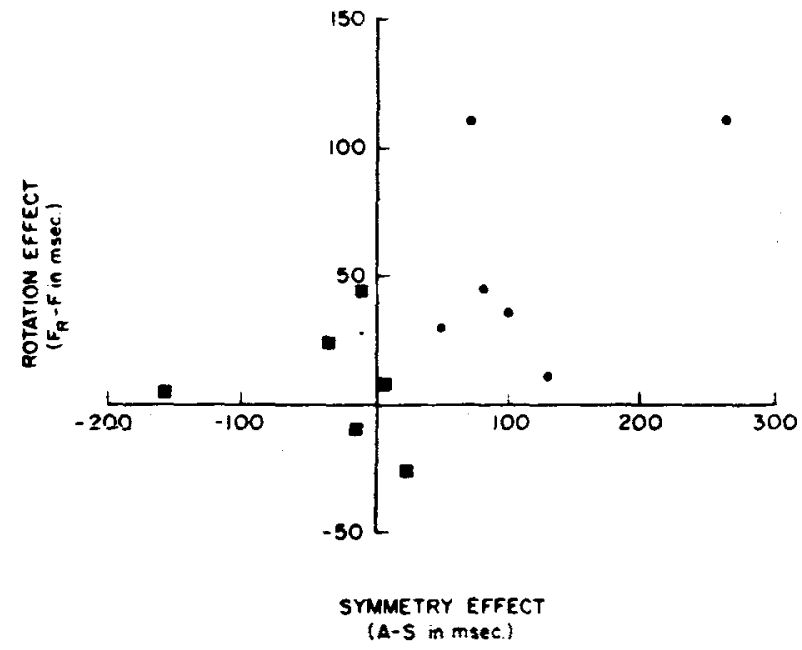

Fig. 3. Scattergram for Ss assigned to the "same" condition. The circles indicate Ss assigned to Group 1; the squares indicate Ss assigned to Group 2.

symmetry effect for the Ss in the "same" condition was that some of these Ss may have adopted the analytic mode of processing hypothesized for "different," but not "same," responses. That is, they may have responded "same" on the basis of matching features rather than comparing the patterns in the wholistic manner hypothesized for structural processes. A correlational analysis was therefore performed to determine whether individual Ss differed with respect to their mode of processing for "same" comparison. For those Ss in the "same" condition who processed the stimulus information in the analytic manner previously hypothesized for only the "different" condition, "same", reaction time should have been unaffected by either symmetry or the rotation of the familiar patterns. That is, their data should be similar to that obtained for Ss who were actually in the "different" condition. For those Ss in the "same" condition who emphasized structural processes, "same" reaction times should have been affected by both symmetry (as originally hypothesized) and rotation (as in Egeth and Blecker's study). The effects of symmetry $(\mathbf{A}-\mathbf{S})$ and rotation $\left(F_{R}-F\right)$ were determined for each $S$, and the correlation of these effects between Ss was computed. ${ }^{3}$ The correlation for the "same" condition $(\mathrm{r}=0.60)$ was significant at the .05 level (the scatterplot for this correlation is presented in Fig. 3). ${ }^{4}$ The correlation obtained for the "different" condition $(r=0.14)$ was insignificant.

The significant correlation obtained for "same" responses provided an explanation for the insignificance of the symmetry effect, since it indicated that the size of the symmetry effect $(A-S)$ had varied between Ss in a systematic rather than a random manner. This necessarily introduced systematic variance into the supposedly random error term used to test the significance of the symmetry effect. biasing the analysis of variance against finding significance. Given the 
Table 2

"Same" Reaction Times (in Milliseconds) and Total Errors With Ss Divided According to Individual Differences

\begin{tabular}{|c|c|c|c|c|c|c|}
\hline & \multicolumn{2}{|c|}{ Mean RT } & \multirow[b]{2}{*}{$(A+S) / 2$} & \multirow[b]{2}{*}{$A-S$} & \multicolumn{2}{|c|}{ Total Errors } \\
\hline & S & $\mathbf{A}$ & & & $\mathbf{S}$ & $\mathbf{A}$ \\
\hline & & & oup $1(\mathrm{St}$ & ctural & & \\
\hline \multirow[t]{3}{*}{$\begin{array}{l}\mathrm{F} \\
\mathrm{F}_{\mathrm{R}}\end{array}$} & $\begin{array}{l}856 \\
914 \\
943\end{array}$ & $\begin{array}{r}969 \\
1031 \\
1047\end{array}$ & $\begin{array}{l}913 \\
973 \\
995\end{array}$ & $\begin{array}{l}+113 \\
+117 \\
+104\end{array}$ & $\begin{array}{l}4 \\
5^{*} \\
6\end{array}$ & $\begin{array}{l}5 \\
3 \\
1\end{array}$ \\
\hline & 904 & 1016 & 960 & +111 & & \\
\hline & \multicolumn{6}{|c|}{ Group 2 (Analytic Ss) } \\
\hline \multirow[t]{2}{*}{$\begin{array}{l}F_{\mathrm{R}} \\
\mathrm{F}_{\mathrm{R}}\end{array}$} & $\begin{array}{l}870 \\
862 \\
940\end{array}$ & $\begin{array}{l}830 \\
853 \\
903\end{array}$ & $\begin{array}{l}850 \\
858 \\
922\end{array}$ & $\begin{array}{r}-40 \\
-9 \\
-37\end{array}$ & $\begin{array}{l}7 \\
2 \\
2\end{array}$ & $\begin{array}{r}10 \\
7 \\
1\end{array}$ \\
\hline & 891 & 862 & 877 & -29 & & \\
\hline
\end{tabular}

*Indicates that an error of omission is included.

in appropriateness of analysis of variance when systematic individual differences are obtained, the conclusion that the effect of symmetry $(A-S)$ on "same" reaction time was not the result of chance variation must rest on the significance of its correlation with the rotation effect.

In order to further examine the individual differences obtained for "same" responses, the Ss in the "same" condition were divided into two groups on the basis of the size of the symmetry effect $(A-S)$ for each $S$. Those six $S s$ with the largest values of $(A-S)$ constituted Group 1; since their data conformed to what was hypothesized for Ss in the "same" condition, these Ss were labeled as emphasizing structural processes. The six Ss with the smallest values of $(A-S)$ constituted Group 2; since their data closely resembled that of the "different" condition, these Ss were labeled as emphasizing analytic processes. Table 2 contains the mean reaction times and errors for the two groups.

An examination of Table 2, which was based solely on "same" responses, indicated that familiarity (F vs U) affected both structural and analytic Ss. ${ }^{5}$ For the structural Ss of Group 1, response times for the rotated-familiar patterns $\left(\mathbf{F}_{\mathbf{R}}\right)$ were almost as slow as response times for the unfamiliar $(\mathrm{U})$ patterns, indicating that the effects of past experience were almost eliminated by the rotational transformation. In addition, the effects of -symmetry and familiarity were clearly additive for the structural Ss. Finally, a comparison of Groups 1 and 2 indicated that the analytic Ss had faster overall reaction times than did the structural Ss. This increase in speed, however, may have been obtained at the cost of reduced accuracy, since overall reaction time and errors of comission were correlated $(\mathrm{r}=0.57$, $\mathrm{p}<.05$ ) between all the Ss in the "same" condition. There was, however, no indication that speed-accuracy tradeoff influenced the familiarity or symmetry effects obtained for either the structural or analytic Ss. ${ }^{6}$

\section{DISCUSSION}

The results of this study supported the two-process hypothesis for same-different comparison, but with the unexpected finding of individual differences in "same" comparison. The absence of a symmetry effect for "different" responses supported the hypothesis that "different" comparison is based on analytic processes. For "same" responses, symmetry affected reaction times for some Ss (Group 1) but not others (Group 2). Since the symmetry effect obtained for Group 1 conformed with the hypothesis for "same" responses, these Ss were said to emphasize structural processes. Since their data closely resembled that of the "different" condition, the Ss in Group 2 of the "same" condition were said to emphasize analytic processes. These individual differences were self-selected by the Ss in the "same" condition; there were no task or instructional manipulations by the $\mathrm{E}$.

\section{Analytic Processes}

The analytic Ss in the "same" condition (same-analytic), like the analytic Ss in the "different" condition (different-analytic), were unaffected by either symmetry or the 180-deg rotation of the familiar patterns into an unfamiliar orientation. In addition, familiarity ( $F$ vs $U$ ) was found to affect reaction times for both same-analytic and different-analytic Ss. This familiarity effect had two important implications. First, it showed that the absence of both symmetry and rotation effects for these Ss was not the artifactual result of their insensitivity to the $\mathrm{E}$ demand implicit in both the manipulation of symmetry and the rotation of the patterns. If this were the case, the analytic Ss would have been similarly insensitive to the E's manipulation of familiarity ( $F$ vs $U$ ). In addition to ruling out the possibility of artifact, the familiarity effect obtained for both the same-analytic and different-analytic Ss suggested that these Ss had acquired knowledge of the distinctive features of the familiar patterns. Furthermore, the absence of a rotation effect $\left(F_{R}-F\right)$ for the analytic Ss suggested that at least some of the distinctive features were rotationally invariant.

\section{Structural Processes}

Structural Ss, so labeled because their "same" responses were affected by symmetry, were also affected by the 180-deg rotation of the familiar patterns into an unfamiliar orientation. The additional finding that response times were almost as slow for the rotated-familiar patterns as for the unfamiliar patterns indicated that the effect of familiarity on structural processes was virtually eliminated by rotational transformation. This finding was not necessarily contradicted by the fact that the structural property of symmetry is rotationally invariant. It may be that rotationally invariant properties (symmetry) are easy to extract, requiring little or no familiarity, while orientation-specific structural properties can be extracted only after the $\mathrm{S}$ has become familiar with the 
stimulus. It is also possible that familiarity involves abstract rules that are qualitatively different from the rules based on stimulus structure (symmetry). Both of these possibilities are compatible with the finding, for structural Ss, that familiarity and symmetry affect "same" reaction time in an additive manner.

In the present study, it has been assumed that symmetry influences structural processes by providing a simple rule for structuring or organizing the detailed parts of a stimulus into a well-formed whole. An alternative way of interpreting the symmetry effect would have Ss use symmetry, not as a rule of organization, but as a rule for reducing the amount of information that must be compared in order to make a "same" response. Thus, the individual differences obtained for the "same" condition could be attributed to whether or not Ss search for redundancy in the stimulus information. Although this alternative was a plausible interpretation of the results of the present study, it is not clear how it would apply to experiments in which pattern redundancy, if present, is not well defined (e.g., the detection of embedded figures ${ }^{7}$ ).

\section{Structural vs Analytic Processes}

\section{Recognition}

The effect of rotation on recognition has been investigated since Dearborn's (1899) original study. This research has indicated that, although recognition is reduced by the rotation of familiar stimuli, the loss is only partial. A partial effect of rotating familiar stimuli was also obtained for the combined data from all the Ss in the "same" condition (Table 1). The evidence for individual differences among these Ss, however, suggested that the effect of rotation on recognition may be an all-or-none phenomenon: "all" for structural Ss and "none" for analytic Ss. The partial reduction in recognition often obtained would then be the artifactual result of combining the data of $\mathrm{Ss}$ who emphasize different modes of processing.

\section{"Same" vs "Different" Responses}

It was found for the "same" condition that Ss emphasizing analytic processes tended to respond more rapidly than did Ss emphasizing structural processes. As discussed in the introduction, the relative speeds of structural and analytic processes may be due to the particular stimuli that were used. It is conceivable that if each stimulus had more connectivity than dot patterns, and the total set of stimuli were less discriminable than the patterns used in this experiment, then structural Ss might have exhibited faster "same" responses than analytic Ss ("same" responses would then be faster than "different" responses, since "same" reaction time would include the responses for structural as well as analytic Ss). If, however, analytic processes are always faster than structural processes, regardless of the stimuli, the question of why "same" responses are sometimes faster than "different" responses would remain unanswered.

\section{Cognitive Controls}

Most recent interest in individual differences in perception has been confined to the investigation of cognitive controls, which involve the slowly changing, situationally invariant relationship between cognitive processes and personality variables (Gardner et al, 1959). Several interesting dimensions of individual difference have been discovered by these investigators. Witkin et al (1954) have distinguished between field-dependent and field-independent $\mathrm{Ss}$ on the basis of their ability to ignore irrelevant cues, and Holzman and Klein (1954) differentiated between levelers and sharpeners on the basis of their tendency to accentuate differences. The relationship between these individual differences and those obtained in the present study, is a question requiring further investigation.

\section{Methodological Possibilities}

Although individual differences were not anticipated at the start of this study, the correlational or individual differences approach seems to be a promising methodology for studying perceptual processes. In tasks where alternative processes seem feasible, the typical approach has been to design experiments for which the results would favor one alternative over the other. The correlational approach would permit the formulation of dual hypotheses corresponding to the two alternative processes. By examining individual differences in performance, it would then be possible to obtain evidence that would provide simultaneous support for both hypotheses. It is anticipated that this methodology will be of value in the further investigations of the individual differences in structural vs analytic processes obtained in this study.

\section{REFERENCES}

Attneave. F. Symmetry, information and memory for patterns. American Journal of Psychology, 1955, 68, 209-222.

Bamber. D. Reaction times and error rates for "same"."different" judgments of multidimensional stimuli. Perception \& Psychophysics, 1969. 6, 169-174.

Beller, H. K. Parallel and serial stages in matching. Journal of Experimental Psychology, 1970.84. 213-219.

Dearborn. G. V. N. Recognition under objective reversal. Psychological Review: 1899. 6. 395-406.

Egeth, H.. \& Blecker, D. Differential effects of familiarity on judgments of sameness and difference. Perception \& Psychophysics. 1971.9. 321-326.

Gardner. R. W.. Holzman. P. S.. Klein, G. S.. Linton. H. B.. \& Spence. D. P. Cognitive control. In Psychological isstes. New York: International University Press. 1959.

Garner. W. R.. \& Clement. D. E. Goodness of pattern and pattern uncertainty. Journal of Verbal Learning \& Verbal Behavior. 1963. 2, 446-452. 
Gibson. J. J. The senses considered as perceptual sistems. New York: Houghton Mifflin, 1966.

Hawkins. H. L. Parallel processing in complex visual discrimination. Perception \& Psychophysics. 1969. 5. 56-64.

Hock. H. S. The role of structure in the perception of familiar and unfamiliar stimuli. Unpublished doctoral dissertation, Johns Hopkins University, 1971.

Holzman, P. S., \& Klein, G. S. Cognitive system principles of leveling and sharpening: Individual differences in assimilation effects in visual time-error. Journal of Psychology, 1954, 37, 105-122.

Krueger, L: E. Effect : of bracketing lines, on speed of "same"-"different" judgment of two adjacent letters. Journal of Experimental Psychology, 1970, 84, 324-330.

IN Nemar. Q. Psychological statistics. New York: Wiley, 1962.

Nickerson. R. S. Binary classification reaction time: A review of some studies of human information processing capabilities. Psychonomic Monograph Supplements, 1972, 4(17, Whole No. 65).

Witkin. H. A., Lewis, A. B., Hertzman, M., Machover, M., Meissner. P. B., \& Wapner, S. Personality through perception An experimental and clinical study. New York: Harper, 1954.

\section{NOTES}

1. The predicted symmetry effect could be based on other assumptions regarding structural processes. An adherent of Gibson's (1966) theory, for example, might argue that symmetry would affect structural processes in that larger chunks of stimulus information could be extracted from symmetrical than from asymmetrical stimuli. Regardless of the assumptions, however, the precise relationship between structural processes and reaction time remains somewhat indeterminate. That is, symmetry might result in faster "same" responses for two different reasons: (1) structural processes could be completed more rapidly for symmetrical than for asymmetrical stimuli, and (2) structural processes could result in a more wholistic percept (fewer chunks) as a result of symmetry, and having to compare "fewer chunks" would make "same" comparison faster for symmetrical stimuli. At present, there is no way of distinguishing between these two alternatives.
2. A diagonal axis of symmetry was necessary in order to generate a sufficient number of symmetrical stimuli.

3 . Although the symmetry effect $(A-S)$ and the rotation effect $\left(F_{R}-F\right)$ include common components (e.g., the familiar-symmetric condition), $(A-S)$ and $\left(F_{R}-F\right)$ are logically orthogonal. In this way, the proposed correlational analysis is based on the same assumptions of statistical independence that are ordinarily applied to analysis of variance with factorial designs.

4. The possibility existed that the obtained correlation was an artifact of performance level. That is, some Ss may have had small symmetry $(A-S)$ as well as small familiarity $\left(F_{R}-F\right)$ effects, because they were responding at a rate of speed close to optimum performance. For $\mathrm{Ss}$ with nonoptimal performance, slower reaction times could have resulted in proportional increases in both $(A-S)$ and $\left(F_{R}-F\right)$, producing an artifactual correlation. A partial correlation coefficient (McNemar, 1962, p. 166) was therefore computed, with performance level, as defined by the reaction time of each $S$ to the familiar, symmetrical patterns, "partialled out." The resulting correlation between $(A-S)$ and $\left(F_{R}-F\right)$ was again 0.60 , ruling out the possibility of artifact.

5. This familiarity effect was not predetermined by the correlational findings [it was plausible that familiarity ( $F$ vs $U$ ) would have no effect on the "same" reaction times of analytic Ss]. Although the size of the familiarity effect was substantial, the number of $S s$ in each group was too small to provide enough power for the differences to be statistically significant.

6 . The results for the "same" condition, including all aspects of the findings with respect to individual differences, have been replicated as part of a study by Hock and Ross (in preparation).

7. Hock, Gordon, and Marcus (in preparation) have found that Ss emphasizing analytic processes detect embedded figures more rapidly than Ss emphasizing structural Ss.

(Received for publication October 5, 1972;

first revision received February 12, 1973; second revision received May 25, 1973.) 